\title{
Miniaturized Ring Resonator Wideband Bandpass Filter with Wide Stop Band
}

\author{
Inder Pal Singh ${ }^{1}$, Praveen Bhatt ${ }^{2}$ \\ ${ }^{1}$ Banasthali University, Banasthali - 304022, India \\ ${ }^{2}$ Asia Pacific Institute of Information and Technology, Faridpur Road, G.T. Road Karnal, Panipat-132103 Haryana, India
}

\begin{tabular}{l}
\hline \hline Article Info \\
\hline Article history: \\
Received Jan 6, 2019 \\
Revised Apr 30, 2019 \\
Accepted Nov 25, 2019 \\
\hline
\end{tabular}

\section{Keyword:}

bandpass filter

stop-band

skirt-characteristics

bandwidth

resonator

\begin{abstract}
In this paper a miniaturized quarter wavelength rectangular shaped multimode ring resonator bandpass filter with extended diagonal corners and internally located high impedance perturbation stubs is proposed. Input/output open stubs are tightly coupled to the extended diagonal corners running parallel to the two opposite sides of the ring resonator, generate wide passband and wide stop-band. Cut-off frequencies can be shifted to the higher side by increasing the length of the sides of the resonator. By inserting the perturbation stubs, rectangular ring resonator produces three degenerate modes out of which first two form a wide passband. Small square patch is attached to the opposite interior corners of the ring resonator to improve the return loss. T-shaped stub is tightly coupled between the feeder line and longer side of the resonator to improve the insertion loss and skirt-characteristics. Shorter opposite sides of the rectangular ring resonator are bent in U-shaped to increase the effective electrical length of the resonator eventually the bandwidth is widened. Filter is designed and simulated for the center frequency of $3.2 \mathrm{GHz}$, bandwidth from $2.0 \mathrm{GHz}$ to $4.0 \mathrm{GHz}$, on dielectric constant 3.38 and thickness $0.508 \mathrm{~mm}$. Electromagnetic simulator Ansoft HFSS is used to optimize the filter dimensions.
\end{abstract}

Copyright $(2019$ Institute of Advanced Engineering and Science. All rights reserved.

\section{Corresponding Author:}

Inder Pal Singh,

Banasthali University,

Banasthali - 304022, India

Email: ipsinghphys@gmail.com

\section{INTRODUCTION}

Microstrip wideband bandpass filter is an important RF end component in wireless communication systems. Federal Communications Commission (FCC) in 2002 allowed unlicensed bandwidth in which the devices can operate at free of cost, but the downside is this opportunity that it has higher signal-to-noise ratio and highly vunerable to interference [1]. In modern era multi-band, multi-mode, ultra-wide-band (UWB) and compact wireless microwave communication handheld devices are in great demand due to low cost, small size and improved performance. Unlicensed UWB spectrum $(3.1 \mathrm{GHz}$ to $10.6 \mathrm{GHz})$ has been gradually over crowded because of low powered data transfer speed is higher and multiple devices are integrating into single compact device therefore it is highly challenging task for the design researchers to design miniaturized, low losses, multi-band, interference tolerance integrated devices. Filtering devices and trans-receivers antenna should be reasonably compact sized for the integrated devices. Numerous design techniques, methods and design topologies have been implemented from a long time to overcome this issue. In [2], authors used three techniques to reduce the dimension of filter. First, decrease the number of resonators. In this design, two U shape resonators are used. Second, used via ground holes technique. And third, use substrate that has higher dielectrict constant. One of the popular design topologies, square ring resonator is used in [3] to design compact ultra-wideband bandpass filter in which two sets of open stubs bent at 900 are running parallelly outside the square ring resonator and one end of open stubs connected to the opposite diagonal corner of the resonator. Metamaterial-based resonators (MBRs) integrated with the defected ground structure (DGS) can effectively improves the coupling excitations to realize wideband bandpass filter. Incorporating a DGS structure in the 
main design reduces the size of the filter and makes it easy to fabricate under the fabrication limit [4]. Three U-shaped multi-mode resonators are tightly coupled in a series to implement ultra-wideband bandpass filter. Circular ring is shunted by high impedance open stub at the base of U-shaped resonator. Resonant modes of this resonator are allocated by adjusting the radius of the ring, length of the side of the resonator and coupling gap between the resonators [5]. In [6], UWB bandpass filter is designed with three resonant modes in multimode-resonator based on half-mode substrate integrated structure and four resonant modes are achieved from a pair of electromagnetic band-gap (EBG) incorporated in a pair of parallel coupled lines. This type of structure is compact in size and produced ultra-large stopband. This structure represents a higher order of selectivity of the passband, this property of the filter increases the tolerance of the design which is highly requires if the design size is too small. In [7] $3 \lambda / 4$ wavelength resonator is presented as an ultra-wideband bandpass filter. One stepped-impedance shorted-stub is perpendicularly attached to one of the square shaped resonator line.

Multimode resonators are widely used in order to miniaturize the filter. The tripe-mode stub-loaded resonator is constructed by a high impedance line with length of 211 and width of w loaded with one folded stepped-impedance open-circuited stub denoted by lengths $(13,214)$ and widths $(2 \mathrm{w}, \mathrm{w})$ and one short-circuited stub with length 12 and width $2 \mathrm{w}$ at the central plane [8]. Compact ultra-wideband bandpass filter is composed of multi-mode resonator (MMR). In this filter three circular shaped patches connected to high impedance open stub are serving as three resonators. Out of three circular patches, two are connected to small square patch through a high impedance stub. This arrangement of circular patches are shunted across the high impedance line. This high impedance line is inserted into two parallel lines which are closely coupled, forming inter-digital lines at input and output port [9]. Stepped impedance resonators (SIR) are one way to dramatically reduce size and miniaturization of microstrip dual band bandpass filters for WLAN applications. T-shaped SIR is used for simplifying the analysis of the circuits [10]. Ultra-wideband from $4.0 \mathrm{GHz}$ to $11.0 \mathrm{GHz}$, fractional bandwidth (FBW) $93.3 \%$ and five transmission poles (TP) in the passband are occurred in miniaturized bandpass filter. Bandpass filter consists two sets of four parallel coupled lines at input and output port. In the middle of these sets of parallel lines two T-shaped stepped impedance lines are placed vertically. Rectangular DGS is placed in the middle of the filter with two other DGSs on either side of it. By introducing the DGSs the filter size is reduced. Arrow coupled lines and U-slot defected ground structures (U-DGS) is proposed and succeeded to reduce the size [11-13]. T-shaped resonator filter is proposed for ultra-wideband bandpass filter. The bandpass filter is composed of four short-circuited stubs of length $\lambda / 4$, where $\lambda$ is the guided wavelength. Filter is miniaturized by implementing the folded stubs instead of keeping it straight, which improves wide passband from $2.4 \mathrm{GHz}$ to $10.9 \mathrm{GHz}$. In this filter T-shaped resonator introduce the notch frequency in the passband [14]. Some researchers emphasis on wide stopband instead of wide passband. A compact and miniaturized microstrip band-stop filter was built by using Rectangular SRR unit cell [15]. By introducing of $\lambda / 4$ stepped impedance resonators, interdigital capacitors and suspended micro-strips, compact bandpass filter in C-band is proposed. $\lambda / 4$ stepped impedance resonator has a unique feature of miniaturization of the filter by controlling the high and low impedance ratios and electrical length of the resonator. Generally, the resonant condition depends upon the electrical length and harmonic frequencies. Demerit of using $\lambda / 4$ stepped impedance resonator is small Q value, so to compensate this problem, suspended micro-strip lines are incorporated in the resonator [16]. Multi-mode resonator in-general is selected in order to design ultra-wide passband compact bandpass filter. Modified multi-mode resonator is proposed to design compact UWB bandpass filter. In an unmodified resonator straight, inter-digital stubs are located at the input and output ports. Size of the filter is $22.5 \mathrm{~mm}$ x $6.5 \mathrm{~mm}$ before modification. Designer bent the parallel coupled lines of inter-digital system in the form of meander pattern. Eventually impedance matching is disturbed because mutual coupling is altered. So, in order to compensate this change, a square patch is attached in middle line of inter-digital system. It is worth mentioning that open-circuited and short-circuited stubs at input and output ports are also responsible for improving the impedance matching in the passband and selectivity of BPF to some extent. After the modification size of filter is reduced to $14.0 \mathrm{~mm}$ x $5.9 \mathrm{~mm}$ so almost successfully $40 \%$ size reduction is achieved [17].

\section{RESEARCH METHOD}

\subsection{Analysis of rectangular Ring Resonator}

Ring resonator of any shape has been used in the past to design wideband bandpass filters. Among all the shapes, square or rectangular ring resonator has few advantages over the other shapes such as small size, low loss and fabrication feasibility. A complex coupling technique to couple input-output lines is used in [18], in this paper complex transmission theory is used to evaluate the resonator parameters and coupling coefficients. In Fig. 1 rectangular shaped ring resonator with two right angled open stubs connected at opposite diagonal vertex of the resonator is shown. This right-angled stub is positioned in such a way that it is running parallel outside the two adjacent sides of the rectangular resonator. Input and output stubs are placed parallel to the longer side of rectangular resonator. Electrical length of four stubs is represented by $\theta_{1}, \theta_{2}, \theta_{3}, \theta_{4}$ and 
corresponding physical lengths $\mathrm{L}_{1}, \mathrm{~L}_{2}, \mathrm{~L}_{3}, \mathrm{~L}_{4} . \mathrm{Z}_{1}$ and $\mathrm{Z}_{2}$ are the impedances of right-angled stub and ring resonator respectively. In Fig. 2 the circuit is similar to Fig. 1 but the difference is that in Fig. 2 two stubs are open stubs connected perpendicularly to the each of the shorter side of rectangular resonator and $\theta_{\mathrm{s}}$ is its electrical length and $Z_{s}$ stub impedance. Small square patch of side length $p$ is placed inside at two diagonally opposite corners of the resonator. In Figs.3 and 4, the even and odd mode circuits of basic rectangular ring resonator are shown, respectively.

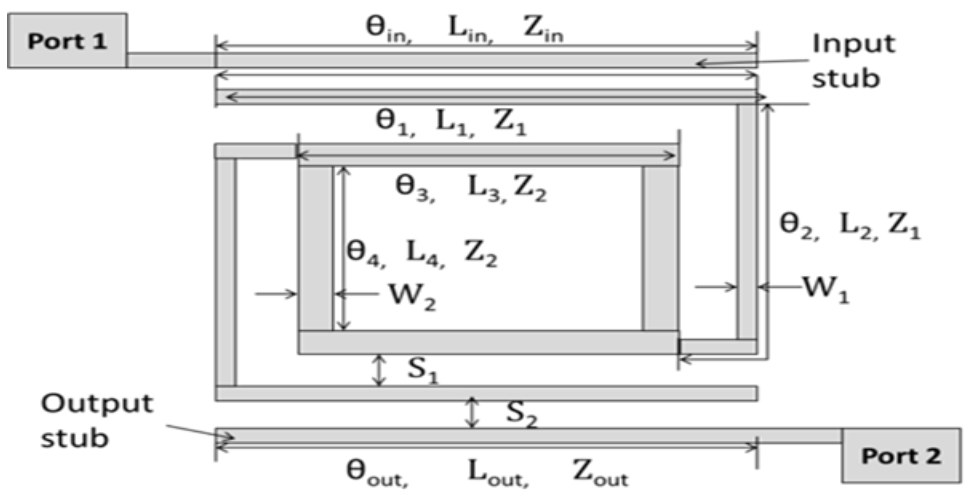

Figure 1. Basic rectangular ring resonator bandpass filter [2].

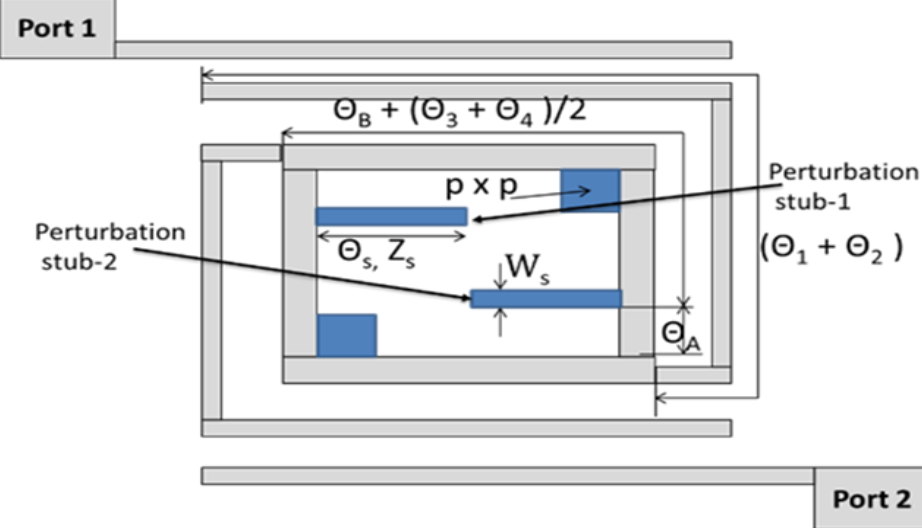

Figure 2. Basic rectangular ring resonator bandpass filter open stubs and square patch [2]

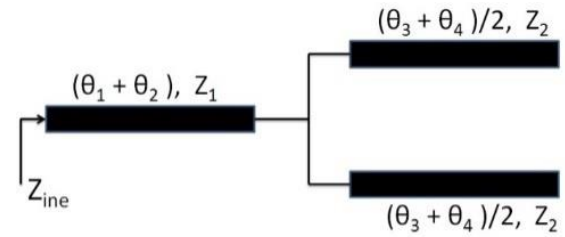

(a)

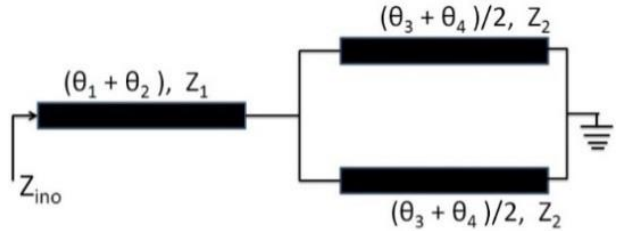

(b)

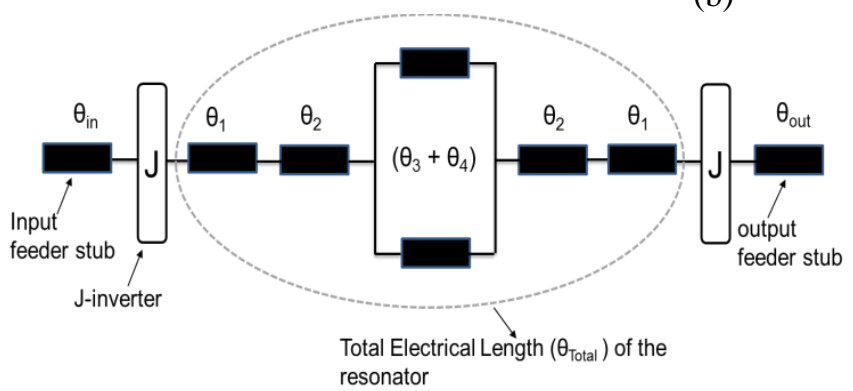

(c)

Figure 3. (a) Even mode circuit model of basic rectangular ring resonator bandpass filter. (b) Odd mode circuit model of basic rectangular ring resonator bandpass filter. (c) Hybrid mode (even + odd) circuit model of total equivalent electrical length of basic rectangular ring resonator incorporated with J-admittance inverter. The condition of resonance of the resonator when it is excited in even mode and odd mode. The inverse of input even and odd impedances $\left(\mathrm{Z}_{\text {ine, }} \mathrm{Z}_{\mathrm{in} 0}\right)$ should be zero at resonant frequencies. 
The resonant conditions are given below:

$$
\begin{array}{ll}
2 R_{Z}+\tan \left(\theta_{1}+\theta_{2}\right) \cdot \cot \left[\left(\theta_{3}+\theta_{4}\right) / 2\right]=0 & \text { even mode } \\
2 R_{Z}-\tan \left(\theta_{1}+\theta_{2}\right) \cdot \tan \left[\left(\theta_{3}+\theta_{4}\right) / 2\right]=0 & \text { odd mode }
\end{array}
$$

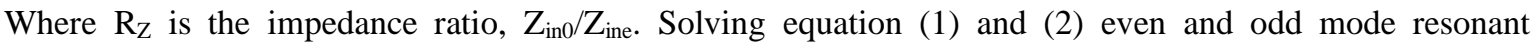
frequencies can be obtained as given below

$$
\begin{aligned}
& f_{\text {odd } 1}=\frac{c}{2\left(2 L_{1}+2 L_{2}+L_{3}+L_{4}\right) \sqrt{\varepsilon_{e f f}}} \\
& f_{\text {even } 1}=\frac{c}{\left(2 L_{1}+2 L_{2}+L_{3}+L_{4}\right) \sqrt{\varepsilon_{\text {eff }}}} \\
& f_{\text {odd } 2}=\frac{3 c}{2\left(2 L_{1}+2 L_{2}+L_{3}+L_{4}\right) \sqrt{\varepsilon_{\text {eff }}}}
\end{aligned}
$$

Where $\mathrm{c}=3 \times 10^{8} \mathrm{~m} / \mathrm{s}, \varepsilon_{\text {eff }}=$ effective dielectric constant of the substrate medium, $\mathrm{L}_{1} \& \mathrm{~L}_{2}=$ physical lengths of low impedance stub, $\mathrm{L}_{3} \& \mathrm{~L}_{4}=$ physical lengths of high impedance stub. For simplicity it is assumed $\mathrm{f}_{\text {odd } 1}=\mathrm{f}_{1}, \mathrm{f}_{\text {even } 1}=\mathrm{f}_{2}, \mathrm{f}_{\mathrm{odd} 2}=\mathrm{f}_{3}$. From the equations (3), (4) and (5) it is clear that $\mathrm{f}_{2}=2 \mathrm{f}_{1}$ and $\mathrm{f}_{3}=3 \mathrm{f}_{1}$. Low impedance stub is denoted by $Z_{1}$, high impedance stub is denoted by $Z_{2}$ and their ratio is denoted by $R_{Z}=Z_{1} /$ $\mathrm{Z}_{2}$.

\subsection{Bandwidth and Stopband of Resonator Filter}

The strong slow-wave effect introduced by the proposed cell, which leads to an ultrawide upperstopband bandpass filtering response [19]. In Fig. 4 two odd mode and one even mode resonant frequency are plotted against the electrical length ratio of low impedance to high impedance stub of the resonator. The value of $R_{Z}$ is fixed 0.5 for the plot. The gap in the frequency $f_{1}$ and $f_{2}$ is a bandwidth and frequency $f_{3}$ is harmonic frequency. As electrical length ratio, $k=\left(\theta_{1}+\theta_{2}\right) /\left(\theta_{3}+\theta_{4}\right)$ increases, gap between $f_{2}$ and $f_{1}$ increases that is bandwidth $\left(f_{2}-f_{1}\right)$ increases. Stopband $\quad\left(f_{3}-f_{2}\right)$ also increases as $k$ increases. At $k=1$ all three frequencies are merged at single value, therefore bandwidth is disappeared.

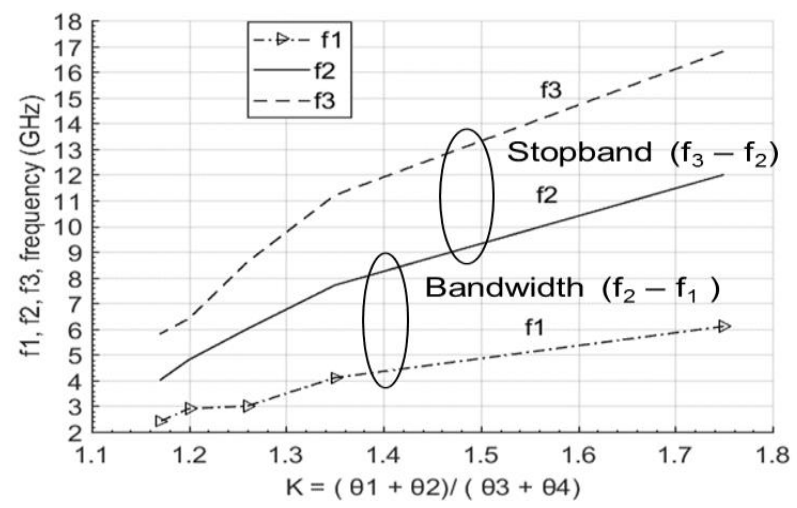

Figure 4. Even and odd mode resonant frequency versus stub length ratio(k).

It is well known that impedance ratio of parallel coupled lines and bandwidth are inversely proportional, apparently bigger impedance ratio $\left(\mathrm{R}_{\mathrm{Z}}\right)$ corresponds to smaller bandwidth. Electrical length ratio (k) is directly proportional to the bandwidth. Small increment in the electrical length ratio generates wide bandwidth at constant impedance ratio. Also, larger electrical length ratio enhances the stopband size. The centre frequency of the passband of the bandpass filter can be given by the equation

$$
f_{c}=\sqrt{\left(f_{1} \cdot f_{2}\right)}
$$

Where, $f_{1}$ and $f_{2}$ are the lower and upper frequency of the passband respectively. It is universly known that, the ratio of the upper and lower frequency $\left(f_{2} / f_{1}\right)$ remains unchanged with respect to the variation in electrical length ratio $(\mathrm{k})$ as long as the impedance ratio $\left(\mathrm{R}_{\mathrm{Z}}\right)$ is fixed. FBW is invariant however electrical length ratio changes. This information provides the degree of freedom for the selection of the $\mathrm{k}$ values eventually precise value of electrical lengths and centre frequency $\left(f_{c}\right)$ can be found.

\subsection{Input-Output coupling}

Resonator filter is coupled between two feeder lines, high impedance stubs, one is input stub and another output stub. It is important to discuss the external quality factor $\left(\mathrm{Q}_{\mathrm{ext}}\right)$ which is given by equation (6) [20].

$$
Q_{\text {ext }}=\frac{\pi}{2} \cdot \frac{1}{\bar{J}^{2}}
$$


Where $\bar{J}=\mathrm{J} / \mathrm{Y}_{\text {in }}$ and $\bar{J}=\mathrm{J} / \mathrm{Y}_{\text {out }}, \mathrm{J}=$ admittance invertor factor, $\mathrm{Y}_{\text {in }}$ and $\mathrm{Y}_{\text {out }}$ are the input and output admittance respectively and $\mathrm{Y}_{\text {in }}=\mathrm{Y}_{\text {out }}$. The value of $\bar{J}$ depends on the factors, $\theta_{\text {in/out }}, Z_{\text {in/out }}, Z_{1}, S_{2}, W_{1}$ so by adjusting these parameters external quality factor is extracted [21].

Fig. 5 depicts the comparison of the impact of two different external quality factors applied on the basic bandpass filter given in Fig. 1. Optimized values are determined to meet the external quality factor for the circuit given in Fig. 1. Two different sets of optimized values are, $\mathrm{L}_{1}=10.4 \mathrm{~mm}, \mathrm{~W}_{1}=0.2 \mathrm{~mm}, \mathrm{~S}_{2}=0.2$ $\mathrm{mm}, \mathrm{S}_{2}=0.4 \mathrm{~mm}$ selected, where in these two sets all values are same except $\mathrm{S}_{2}$. Other parameters of this circuit are chosen, electrical length ratio from Fig. $5, \mathrm{k}=1.2$, dielectric constant $=3.38$, substrate height $=$ $0.508 \mathrm{~mm}$, centre frequency $\left(\mathrm{f}_{\mathrm{c}}\right)=3.2 \mathrm{GHz}$. Low to high Impedance ratio $\left(\mathrm{R}_{\mathrm{z}}\right)$ is 0.5 is selected. The lower value of $S_{2}$ corresponds to the tight coupling and vice-versa.

In case of strong coupling insertion loss is reduced and return loss is also improved. Two transmission poles are occurred within the passband moreover, position of transmission poles in both type of coupling is same.

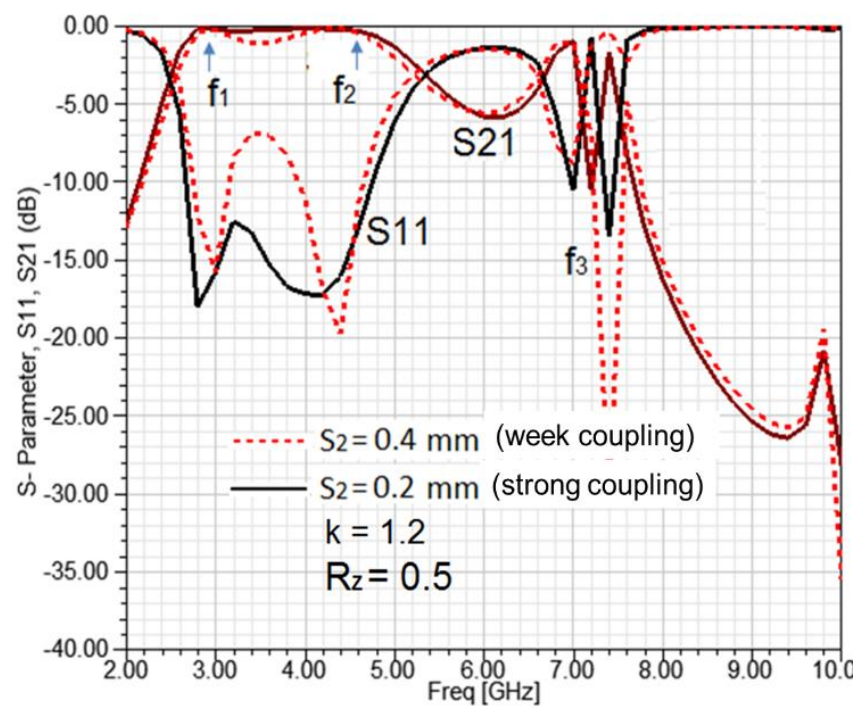

Figure 5. S-Parameter (S11, S21) vs frequency of basic bandpass filter with tight and week input-output coupling. strong coupling gap $\mathrm{S}_{2}=0.2 \mathrm{~mm}$ and week coupling gap $\mathrm{S}_{2}=0.4 \mathrm{~mm}$. Circuit dimension $\mathrm{L}_{1}=10.4$ $\mathrm{mm}, \mathrm{L}_{2}=5.4 \mathrm{~mm}, \mathrm{~L}_{3}=9.2 \mathrm{~mm}, \mathrm{~L}_{4}=4.6 \mathrm{~mm}, \mathrm{~W}_{1}=0.4 \mathrm{~mm}, \mathrm{~W}_{2}=0.2 \mathrm{~mm}, \mathrm{~S}_{1}=0.4 \mathrm{~mm}$.

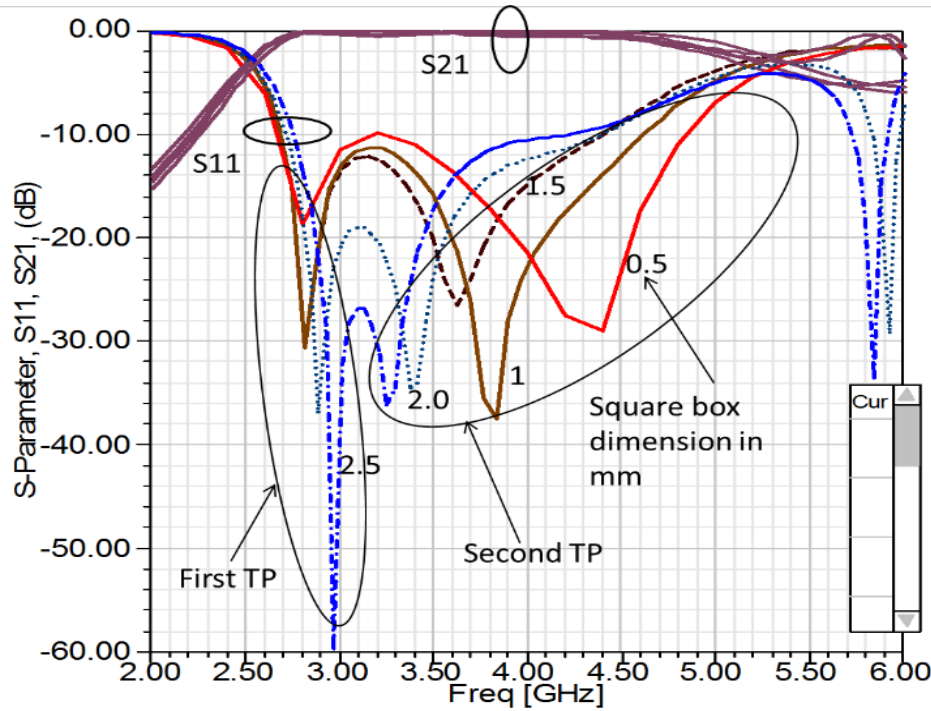

Figure 6. S-parameter response (S11, S21) against the different dimensions of square box, $\mathrm{p}=0.5,1.0,1.5$, $2.0,2.5 \mathrm{~mm}, \mathrm{~S}_{2}=0.2 \mathrm{~mm}$.

In Fig. 6 frequency response is shown with respect to the variation in length of the side (p) of square patch placed diagonally at two corners of the basic rectangular ring resonator shown in Fig. 2. Inserting the 
square box of dimension $\mathrm{p}$ in resonator doesn't alter the bandwidth of the resonator but enhances the coupling strength. Two transmission poles (TP) are generated in the passband. No change is observed in the position of all first transmission pole (TP), near to the lower band frequency, with respect to different dimensions of the square patch. The position of all second transmission pole, near to the upper band frequency, is shifted. Five different dimensions of the square patch, from $0.5 \mathrm{~mm}$ to $2.5 \mathrm{~mm}$ are selected for the comparison. As the size of the square patch increases the position of all second TPs are shifted towards the first TP. Therefore, the separation between the first TP and second TP is reduced as the patch size increases. Simultaneously return loss is mitigated. Beyond the threshold size of the patch, these two close transmission poles would be merged and resulted in only single transmission poles. Five different S11 results are obtained with respect to different square patch. It is clear from the graph given in Fig.7 return loss, $-27 \mathrm{~dB}$, is minimum at $\mathrm{p}=2.5 \mathrm{~mm}$.

Return loss is improved by at least $15 \mathrm{~dB}$ by inserting the square patch. By selecting the optimum data of patch area, good frequency can be achieved.

\subsection{Resonant Conditions of poles and zeroes}

In both Figs. 5 and 6, two different strategies, i.e. one strong coupling and other inserting square patch in the diagonal corners of resonator are implemented to reduce the return loss and succeeded but the numbers of transmission poles could not be increased, as it is limited to two only. Another problem is third frequency $\left(f_{3}\right)$ is appearing close to second frequency $\left(f_{2}\right)$ hence stopband is squeezed. These two problems can be rectified when a pair of loaded open ended perturbation stubs $\left(\mathrm{d}_{\mathrm{S}}\right)$ are attached perpendicularly to the opposite sides of the resonator and its equivalent even mode and odd mode circuit diagrams are presented in Fig.7 (a) and (b), respectively.

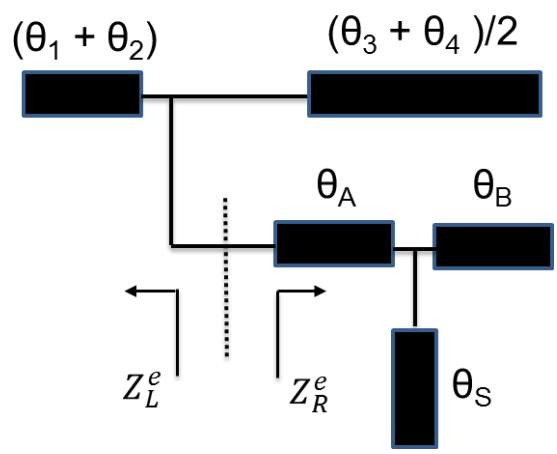

(a)

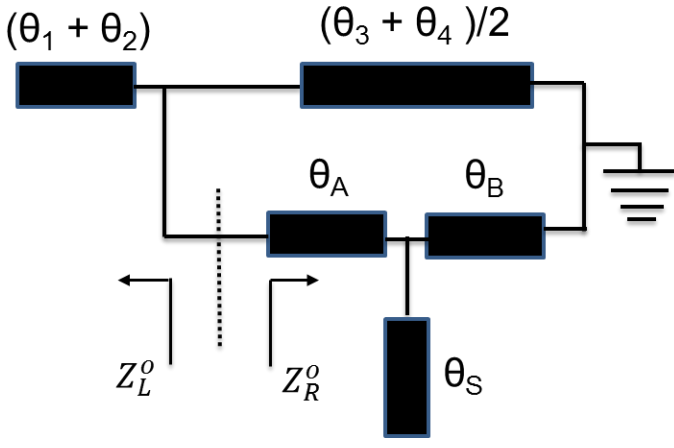

(b)

Figure 7. (a) Even mode circuit model of basic rectangular ring resonator bandpass filter with open perturbation stubs. (b) Odd mode circuit model of basic rectangular ring resonator bandpass filter with open perturbation stubs.

Even mode resonant condition for transmission pole is gieven by equation (8)

$R_{Z} R_{S} \cdot \tan \theta_{B}+R_{Z} \tan \theta_{S}-R_{Z} R_{S} \cdot \tan \theta_{A}+R_{Z} R_{S} \cdot \tan \left(\frac{\theta_{3}+\theta_{4}}{2}\right)+R_{S} \cdot \tan \left(\theta_{1}+\theta_{2}\right)+$

$\tan \theta_{A} \cdot\left(R_{S} \cdot \tan \theta_{B}+\tan \theta_{S}\right) \cdot\left[\tan \left(\theta_{1}+\theta_{2}\right)+R_{Z} \cdot \tan \left(\frac{\theta_{3}+\theta_{4}}{2}\right)\right]=0$

Odd mode resonant condition for transmission pole is given by equation (9)

$\left[R_{Z}-\tan \left(\frac{\theta_{3}+\theta_{4}}{2}\right) \cdot\left(\tan \theta_{1}+\tan \theta_{2}\right)\right] \cdot\left[R_{S} \cdot\left(\tan \theta_{A}+\tan \theta_{A}\right)-\tan \theta_{B} \tan \theta_{S} \tan \theta_{A}\right]=0$

In the above equations $\theta_{\mathrm{A}}$ and $\theta_{\mathrm{S}}$ are the tapping distance and electrical length of perturbation stub respectively. Resonant condition for transmission zero in the stopband is given equation (10) $\cot \theta_{S}=0$

Equation (10) shows the resonant condition of transmission zero when perturbation stub is incorporated in rectangular resonator. Electrical length $\left(\theta_{\mathrm{S}}\right)$ of perturbation stub is quarter wavelength long. Even and odd mode conditions for the passband of the resonator (Fig. 2) when perturbation stubs are attached given by equation (8) and (9). These equations are similar to equation (1) and (2), only difference is that in equations $(8)$ and $(9)$ the tapping distance $\left(\theta_{\mathrm{A}}\right)$ and length $\left(\theta_{\mathrm{A}}\right)$ of perturbation stub is included. 
In Fig. $8 \mathrm{R}_{\mathrm{Z}}=\mathrm{Z}_{1} / \mathrm{Z}_{2}=0.5, \mathrm{R}_{\mathrm{S}}=$ ratio of impedance of perturbation stub-1 to stub-2, $\mathrm{R}_{\mathrm{S}}=\mathrm{Z}_{\mathrm{S} 1} / \mathrm{Z}_{\mathrm{S} 2}=$ 1. Two transmission poles in the passband are identical for two different perturbation stub lengths, $\mathrm{d}_{1}=0 \mathrm{~mm}$ and $\mathrm{d}_{3}=6.0 \mathrm{~mm}$. Transmission zero on right side of passband $\left(\mathrm{f}_{\mathrm{z} 3}\right)$ of $\mathrm{d}_{3}=6.0 \mathrm{~mm}$ is positioned in the vicinity of the passband. Skirt characteristics is improved in case of $\mathrm{d}_{3}$ but no increment in the transmission poles. Transmission zero $\left(\mathrm{f}_{\mathrm{Z} 2}\right)$ of $\mathrm{d}_{2}=4.5 \mathrm{~mm}$ is located away right side from the passband, obviously its skirt is not good but one more transmission pole is added. No significant variation is observed in the lower frequency $\left(\mathrm{f}_{1}\right)$ but marginal change noticed in upper frequency $\left(\mathrm{f}_{2}\right)$ for different stub lengths. Three transmission poles occurred for $\mathrm{d}_{2}$ and two transmission poles for $\mathrm{d}_{1}$. Third transmission pole in $\mathrm{d}_{1}$ is merged into the passband. Third resonant frequency $\left(f_{3}\right)$ is shifted away from the passband when perturbation stub length $\left(d_{S}\right)$ is increased from 0 to $6.0 \mathrm{~mm}$.

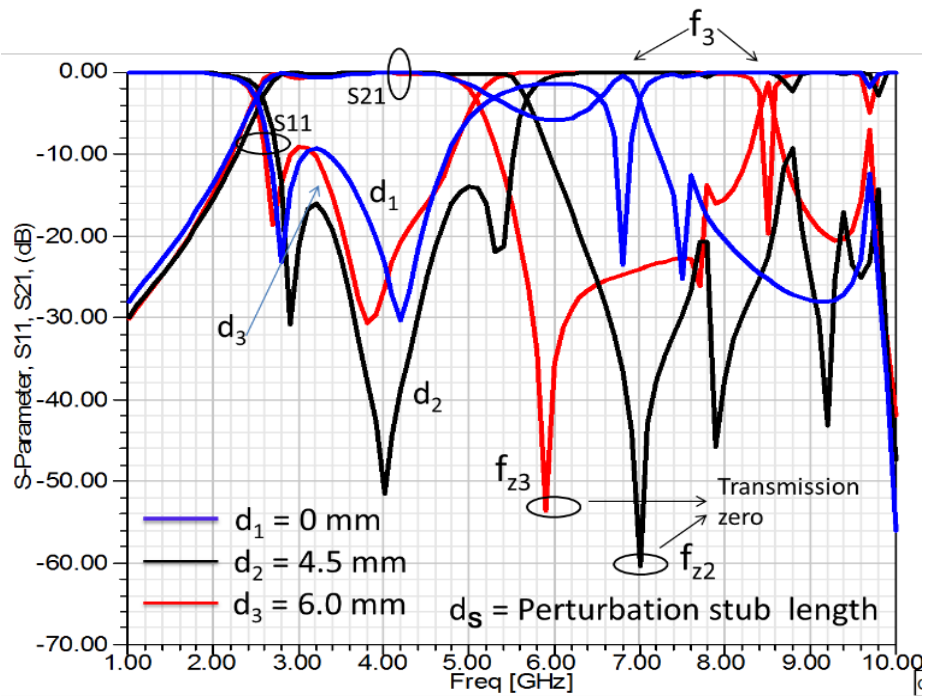

Figure 8. S-parameter response of ring resonator vs different perturbation stubs lengths (ds) attached perpendicularly to the opposite sides of the ring resonator. Dimension of square patch, $\mathrm{p}=0 \mathrm{~mm}$. Circuit dimension $\mathrm{L}_{1}=10.4 \mathrm{~mm}, \mathrm{~L}_{2}=5.4 \mathrm{~mm}, \mathrm{~L}_{3}=9.2 \mathrm{~mm}, \mathrm{~L}_{4}=4.6 \mathrm{~mm}, \mathrm{~W}_{1}=0.4 \mathrm{~mm}, \mathrm{~W}_{2}=0.2 \mathrm{~mm}, \mathrm{~S}_{1}=0.4$ $\mathrm{mm}, \mathrm{S}_{2}=0.2 \mathrm{~mm}$.

\section{Miniaturized Bandpass Filter Design Rules}

Designed values of bandpass filter are centre frequency $\left(\mathrm{f}_{\mathrm{c}}\right)=3.2 \mathrm{GHz}$, bandwidth $=2.0 \mathrm{GHz}, \mathrm{FBW}$ $=62.0 \%$, dielectric constant 3.38, height of substrate $0.508 \mathrm{~mm}$, copper cladding thickness $0.035 \mathrm{~mm}$. To determine the desired value of FBW, first value of $\mathrm{R}_{Z}$ has to be fixed. $\mathrm{R}_{\mathrm{Z}}$ value depends upon the width of the resonator side and width of the input-output feeder stubs which is fixed at $R_{Z}=0.5$. Rectangular resonator is coupled between two parallel feeder stubs. In order to retrieve the desired dimensions of the rectangular resonator, value of $\mathrm{k}=1.22$ is chosen from Fig. 5, regarding this $\mathrm{k}$ value $\Delta \mathrm{f}=\mathrm{f}_{2}-\mathrm{f}_{1}=2.0 \mathrm{GHz}$. For $\mathrm{k}=1.22$, the optimized and simulated values of electrical lengths are given as $\theta_{1}=68.9^{0}, \theta_{2}=45.3^{0}, \theta_{3}=60.7^{0}, \theta_{4}=$ $30.3^{0}, \theta_{\text {in }}=\theta_{\text {out }}=\theta_{1}, \theta_{\mathrm{A}}=5.9^{0}, \theta_{\mathrm{S}}=27.2^{0}$ and their corresponding physical lengths are $\mathrm{L}_{1}=11.4 \mathrm{~mm}, \mathrm{~L}_{2}=6.5$ $\mathrm{mm}, \mathrm{L}_{3}=10.2 \mathrm{~mm}, \mathrm{~L}_{4}=4.86 \mathrm{~mm}, \mathrm{R}_{\mathrm{Z}}=0.5, \mathrm{R}_{\mathrm{S}}=1, \mathrm{~S}_{1}=0.4 \mathrm{~mm}, \mathrm{~S}_{2}=0.2 \mathrm{~mm}, \mathrm{Z}_{\text {in }}=\mathrm{Z}_{\text {out }}=\mathrm{Z}_{1}, \mathrm{~W}_{1}=0.4 \mathrm{~mm}$, $\mathrm{W}_{2}=0.2 \mathrm{~mm}, \mathrm{~L}_{\mathrm{A}}=1.0 \mathrm{~mm}, \mathrm{~d}_{\mathrm{S}}=4.5 \mathrm{~mm}$.

Fig. 9 shows the S-parameter response of basic wideband bandpass resonator filter. It has insertion loss of $1.0 \mathrm{~dB}$, return loss $-8.0 \mathrm{~dB}$, centre frequency $3.2 \mathrm{GHz}$. Symmetrically placed two transmission poles are observed in the passband, first, occurred near to the lower bandpass frequency and second, near to the upper bandpass frequency. It has wide stop-band from $3.8 \mathrm{GHz}$ to 6.4 with two transmission zeros (TZ) at - $46 \mathrm{~dB}$, 4.8 GHz and $-39 \mathrm{~dB}, 6.0 \mathrm{GHz}$. Total area of the filter is $13.0 \mathrm{~mm} \times 7.0 \mathrm{~mm}=91.0 \mathrm{~mm}^{2}$.

Similar to Fig. 9 but improved S-parameter response is replicated by a similar modified filter design with reduced size. Size reduction and optimization of the design parameters has been done by a series of calculation and electromagnetic simulation on HFSS. In Fig. 10 proposed miniaturized wideband bandpass filter is shown. In proposed miniaturized designed, two shorter opposite sides of the rectangular resonator are bent internally in U-shape and other two longer opposite sides are also reduced in length to some extent, but the feeder input-output stubs are increased in length and folded at the corners of rectangular resonators and end of the feeder line is inserted in U-shaped structure and placed exactly in the middle. In miniaturized filter Tshaped structure acts as a coupler. To enhance the input-output coupling high impedance T-shaped stub or a structure is inserted in-between input feeder and the filter and another high impedance T-shaped structure is fed in-between output feeder and the filter. 


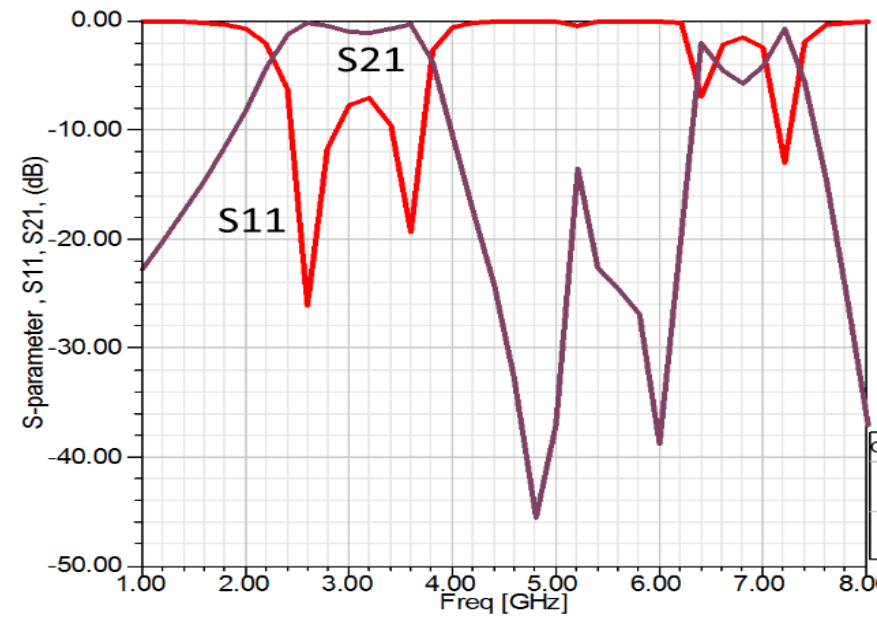

Figure 9. S-parameter response of traditional rectangular shaped resonator bandpass filter. Physical parameter of the filter, $\mathrm{L}_{1}=11.4 \mathrm{~mm}, \mathrm{~L}_{2}=6.5 \mathrm{~mm}, \mathrm{~L}_{3}=10.2 \mathrm{~mm}, \mathrm{~L}_{4}=4.86 \mathrm{~mm}, \mathrm{R}_{\mathrm{Z}}=0.5, \mathrm{R}_{\mathrm{S}}=1, \mathrm{~S}_{1}=0.4 \mathrm{~mm}, \mathrm{~S}_{2}=$ $0.2 \mathrm{~mm}, \mathrm{~W}_{1}=0.4 \mathrm{~mm}, \mathrm{~W}_{2}=0.2 \mathrm{~mm}, \mathrm{~L}_{\mathrm{A}}=1.0 \mathrm{~mm}, \mathrm{~d}_{\mathrm{s}}=4.5 \mathrm{~mm}$.

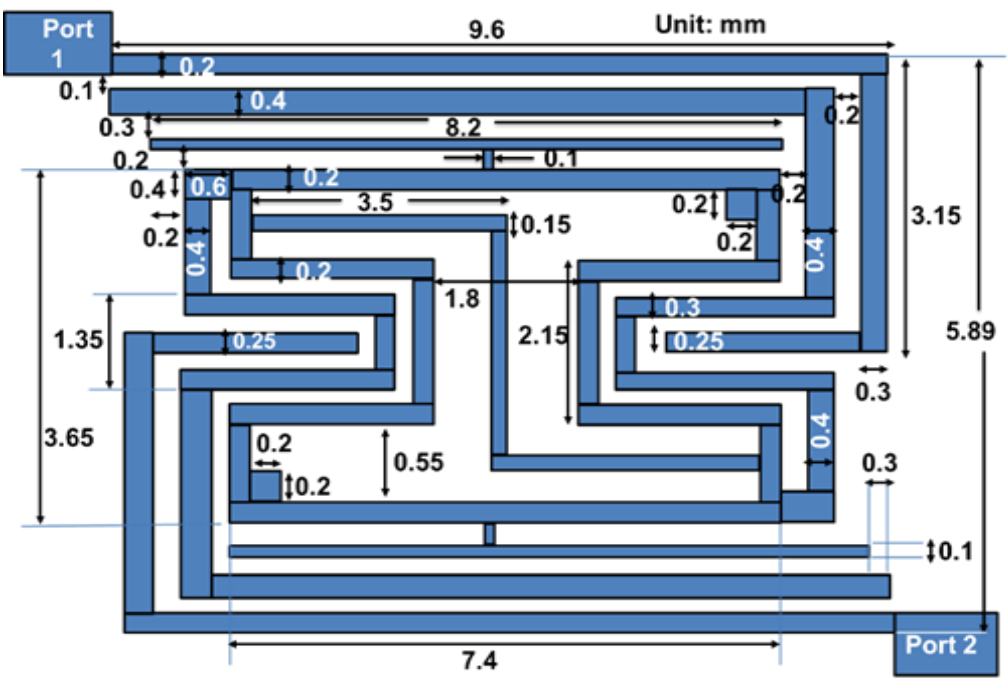

Figure 10. Design layout of miniaturized bandpass filter.

\section{Result and Discussion}

Frequency response of proposed miniaturized bandpass filter is represented by fig. 11. This filter shows $-3 \mathrm{~dB}$ lower and upper frequency of the passband, $\mathrm{f}_{1}=2.1 \mathrm{GHz}$ and $\mathrm{f}_{2}=3.9 \mathrm{GHz}$ at the centre frequency, $\mathrm{f}_{\mathrm{c}}=3.2 \mathrm{GHz}$. Bandwidth, $\Delta \mathrm{f}=\mathrm{f}_{1}-\mathrm{f}_{2}=1.8 \mathrm{GHz}$, fractional bandwidth $(\mathrm{FBW})=53 \%$. Our designed bandwidth is $\Delta \mathrm{f}=2.0 \mathrm{GHz}$. Simulated bandwidth is $\Delta \mathrm{f}=1.8 \mathrm{GHz}$ which is close to the designed value. Return loss is -21 $\mathrm{dB}$ and insertion $0 \mathrm{~dB}$. In the passband two transmission poles are occurred at $-50 \mathrm{~dB}, 2.7 \mathrm{GHz}$ and $-34 \mathrm{~dB}, 3.4$ $\mathrm{GHz}$. Wide 4.0 GHz stopband with two transmission zeros at $6.0 \mathrm{GHz}$ and $7.2 \mathrm{GHz}$ are seen. A good skirt response is obtained.

Two techniques we have implemented here to reduce the return loss without changing the $f_{1}$ and $f_{2}$ values significantly. One method is, increase the coupling between input-output stubs and another introducing the square patch of certain area at the opposite diagonal corners of the resonator. Return loss reduction technique has been mentioned in Fig. 6 and Fig. 7. In order to widen the stopband, two perturbation stubs of unit impedance ratio attached to opposite sides of the resonator.

In Fig. 11, return loss, position of transmission poles (TP) and numbers of TPs are investigated by reflection coefficient (S11) which is given by equation (11)

$S_{11}=\frac{j\left(1-\bar{J}^{4}\right) \cdot \tan \left(\theta_{\text {Total }}\right)}{2 \bar{J}^{4}+j\left(1+\bar{J}^{4}\right) \cdot \tan \left(\theta_{\text {Total }}\right)}$

Where $\bar{J}=\mathrm{J} / \mathrm{Y}_{\text {in }}$ and $\bar{J}=\mathrm{J} / \mathrm{Y}_{\text {out }}, \mathrm{J}=$ admittance invertor factor, $\mathrm{Y}_{\text {in }}$ and $\mathrm{Y}_{\text {out }}$ are the input and output admittance respectively and $\mathrm{Y}_{\text {in }}=\mathrm{Y}_{\text {out }}, \theta_{\text {Total }}=$ total electrical length of the resonator. 
From equation (11) the condition at which return loss is minimum is given as $\left|S_{11}\right|=0$ To meet this requirement the following boundary condition is stated below: $\tan \left(\theta_{\text {Total }}\right)=0$ or $\bar{J}=1$

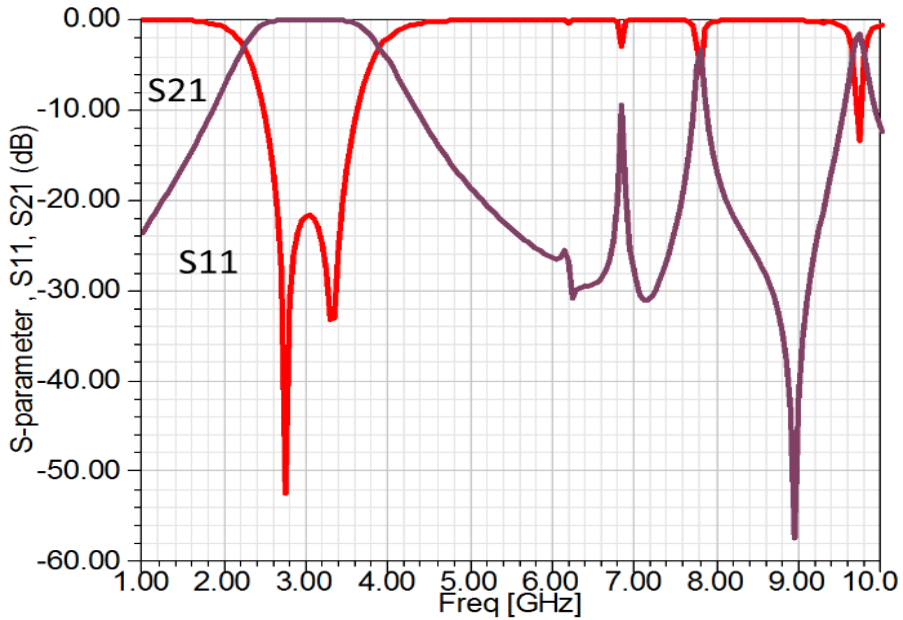

Figure 11. Simulated S - parameter response of miniaturized bandpass filter.

Table 1. Size comparison of bandpass filter

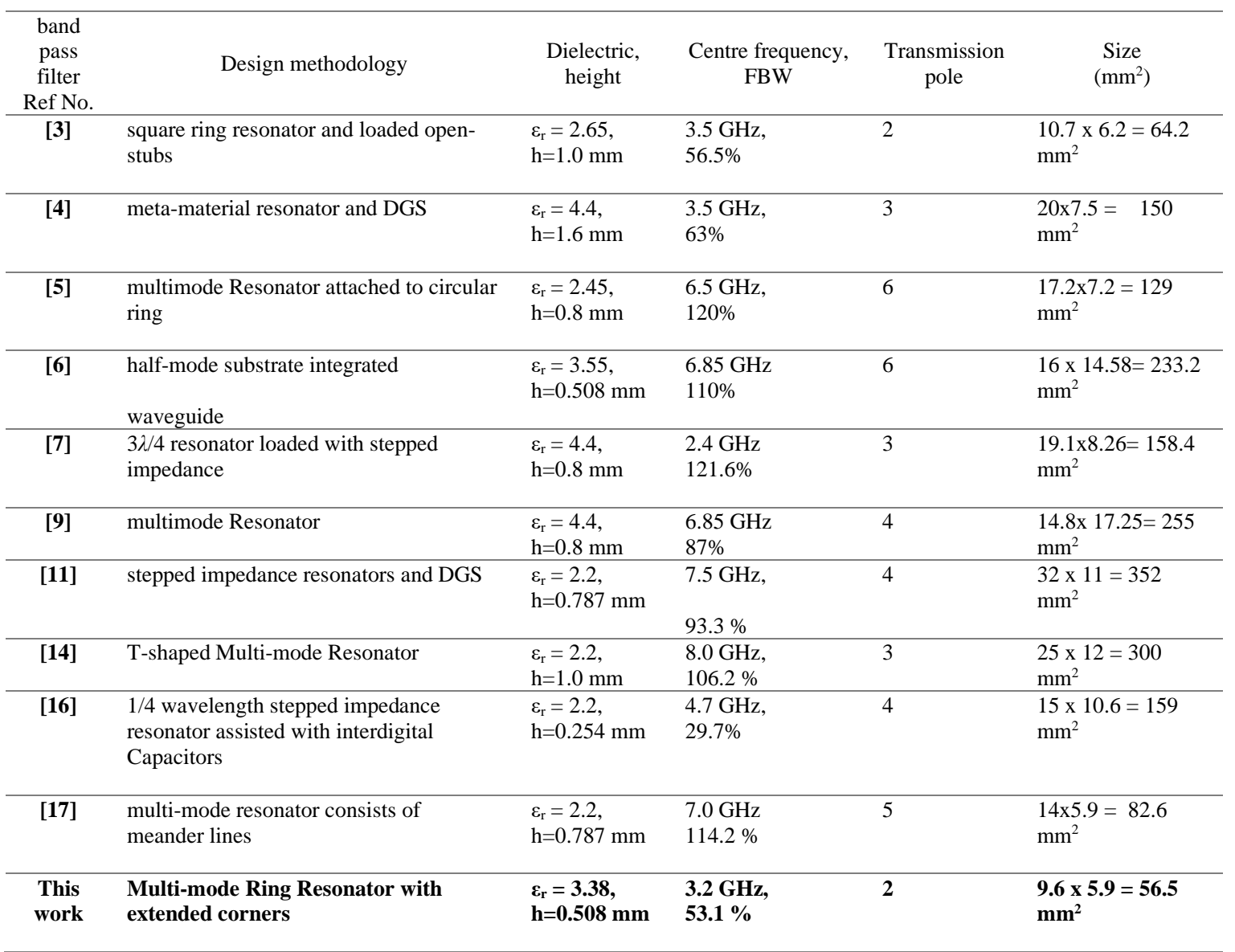

Putting the value of first boundary condition, $\tan \left(\theta_{\text {Total }}\right)=0$, in equation (8) and (9), information about the frequency locations within the passband, where the transmission poles are occurred can be obtained. To apply the second condition of $\mathrm{J}$ - inverter, $\mathrm{J}=1$ in equation (7) to determine the required external quality factor ( $Q_{\text {ext. }}$ ) which is related to input-output feeder electrical length, even and odd impedances which is further determined by line width and the spacing between the feeder line and resonator. In Table. 1, the size of the 
proposed miniaturized bandpass filter is compared with previous research on the similar and traditional design and topology from [3]-[17]. It has been found that our design occupied the least size on the substrate. One of the important factors for the miniaturization of the filter is that we kept the centre frequency value $\left(\mathrm{f}_{\mathrm{C}}=3.2\right.$ $\mathrm{GHz}$ ) below the fundamental frequency $\left(\mathrm{f}_{\mathrm{O}}\right)$ of the bandpass filter resonator, which is close to $8.0 \mathrm{GHz}$. In all the previous research papers centre frequency of the bandpass filter is close to its fundamental frequency which contributes to the larger filter size.

\section{CONCLUSION}

In this paper traditional ring resonator bandpass filter is compared with our modified design. Sparameter response of tradition ring resonator as shown in Fig. 9 has high insertion and return loss, less wide stopband compared to proposed ring resonator bandpass filter. Its return loss is $-8.0 \mathrm{~dB}$ and insertion loss 0.9 $\mathrm{dB}$. Its stopband is $2.4 \mathrm{GHz}$ wide. Area of traditional filter is $91.0 \mathrm{~mm}^{2}$ and proposed filter $56.5 \mathrm{~mm}^{2}$ hence it is $61 \%$ smaller in size. Moreover 10 previously published filter parameters are shown in Table 1 and compared with proposed designed therefore, found that our designed filter occupied least area. In Table 1 the area of the filter which is close to our proposed filter area is given by [3], that is $64.2 \mathrm{~mm}^{2}$ and area of proposed filter 56.5 $\mathrm{mm}^{2}$. In this paper we focused on the miniaturization of the filter instead of improving the performance of the filter to the great extent.

In this paper proposed design of bandpass filter is compared with ten previously published designs specially with their patch area and it is shown in Table 1 that the patch area of the proposed filter is least among all the filters have been discussed.

\section{REFERENCES}

[1] Revision of Part 15 of the Commission's Rules Regarding Ultra-Wideband Transmission Systems, document FCC ET Docket 98-153,2002.

[2] Darwis F, Arief S, Susanti ND. Design of Compact Microstrip U Shape Bandpass Filter Using Via Ground Holes. TELKOMNIKA (Telecommunication Computing Electronics and Control). 2016; 14 (1): 82-85.

[3] Deng K, Chen J Z, Wu B, Su T, Liang C H. Miniaturized wideband bandpass filter utilizing square ring resonator and loaded open-stub. Progress in Electromagnetics Research C 2013; 39:179-192. doi:10.2528/PIERC13032706.

[4] Sarawuth C, Prayoot A. Miniaturized wideband bandpass filter with wide stopband using metamaterial-based resonator and defected ground structure. Radioengineering 2012; 21(2):611-616. DOI: 10.13164/re

[5] Lang Wang, Han-Li Liu, Joshua Le-Wei Li. Novel uwb bandpass filter using multiple-mode resonator units in series. IEEE International Workshop on Electromagnetics: Applications and Student Innovation Competition 2012; 1-2. https://doi.org/10.1109/iwem.2012.6320373

[6] Zhou C X, Guo P P, Zhou K, Wu W. Design of a compact uwb filter with high selectivity and superwide stopband. IEEE Microwave and Wireless Components Letters 2017; 27(7):636-638. https://doi.org/10.1109/lmwc.2017.2711509

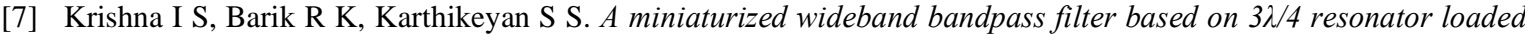
with stepped impedance. Twenty-third National Conference on Communications (NCC), Chennai, 2017;1-5. https://doi.org/10.1109/ncc.2017.8077125

[8] Chen W, Zhao Y, Junof Z. Compact and Wide Upper-Stopband Triple-Mode Broadband Microstrip BPF. TELKOMNIKA (Telecommunication Computing Electronics and Control). 2012; 10(2): 353-358.

[9] Partha P S, Jyoti R P. A compact circular stepped impedance wide-band bandpass filter using multiple-mode resonator. International Conference on Communication and Signal Processing, India 2017; 376-379. https://doi.org/10.1109/iccsp.2017.8286381

[10] Ahmed E. Compact dual-band parallel coupled T-shaped SIR filter for WLAN applications. Telkomnika (Telecommunication Computing Electronics and Control). 2017; 15: 1677-1681. 10.12928/TELKOMNIKA.v15i4.5584.

[11] Hussain I, Das S, Tiary M G. An ultra wideband microstrip bandpass filter with wide stopband using SIR and DGS. 2018 3rd International Conference on Microwave and Photonics (ICMAP), Dhanbad, 2018; 1-2. https://doi.org/10.1109/icmap.2018.8354646

[12] Deena A, Ashraf S, Sebak A. A compact ultra wideband bandpass filter using arrow coupled lines with defected ground structure. Journal of Electrical Systems and Information Technology. 2014; 1(1): 36-44. DOI10.1016/j.jesit.2014.03.001

[13] Hassiba L, Mouloud C, M'hamed B. Compact Ultra-Wide Band Bandpass Filter Design Employing Multiple-Mode Resonator and Defected Ground Structure. The International Conference on Advanced Wireless, Information, and Communication Technologies (AWICT 2015). 2015; 73: 376-383.

[14] Xuemei Z, Wenqi L, Xiaowei Z, Tao J. Design of dual band-notch UWB bandpass filter based on T-shaped resonator. 2016 Progress in Electromagnetic Research Symposium (PIERS), Shanghai, 2016; 4482-4486. https://doi.org/10.1109/piers.2016.7735656

[15] Nasiri B, Errkik A, Zbitou J, Tajmouati A, El Abdellaoui L, Latrach M. A New Compact and Wide-band Band-stop Filter Using Rectangular SRR. TELKOMNIKA (Telecommunication Computing Electronics and Control). 2018; 16(1): 110-117. 
[16] Xuejiao W, Xiaochuan Z, Fei Y, Ting K. Research and design of a miniaturized bandpass filter with ultra-wide stopband. 2015 IEEE International Conference on Communication Problem-Solving (ICCP), Guilin, 2015; 338-340. https://doi.org/10.1109/iccps.2015.7454167

[17] Sahu B, Singh S, Meshram M, Singh S. P. Ultra-compact Microstrip UWB bandpass filter with improved performance. 2017 International Symposium on Antennas and Propagation (ISAP), Phuket, 2017; 1-2. https://doi.org/10.1109/isanp.2017.8228871

[18] Kun D, Shuai Y, Shoujia S, Bian W, Xiaowei S. Dual-mode dual-band bandpass filter based on square loop resonator. Progress In Electromagnetics Research C 2013; 37:119-130. https://doi.org/10.2528/pierc13010605

[19] Ching WT, Li-Ping L, Che-Wei S and Janne-Wha W. Design of dual-and triple-passband bandpass filters with interdigital resonators. IEEE MTT-S International Microwave Symposium Digest, Atlanta, GA, USA, 2018; 539542.

[20] Ma Z W, Sasaki H, Chen C P, Anada T, Kobayashi Y. Design of a wideband bandpass filter using microstrip parallelcoupled dual-mode ring resonator. Asia-Pacific Microwave Conference Proceedings, Dec. 2010; 21-24.

[21] Hong, J.-S. and M.-J. Lancaster, Microstrip Filters for RF/Microwave Applications, John Wiley \& Sons, Inc., 2001. 